\title{
A kind of Supporting Structure of the Thromboelastography
}

\author{
$\mathrm{He} X \mathrm{u}^{\mathrm{a}}$, Fan $\mathrm{Li}^{\mathrm{b}}$, Jianhui Zhao ${ }^{\mathrm{c}}$, Fan $\mathrm{Xu}^{\mathrm{d}}$ \\ School of instrumentation Science and Opto-electronics Engineering, Beihang University, Beijing, \\ 100191, China
}

axuhe200907@163.com, blifan@buaa.edu.cn, 'zhaojianhui@buaa.edu.cn, dx123xufan@163.com

Keywords: thromboelastography, magnetic bearing, active magnetic bearing , passive magnetic bearing.

\begin{abstract}
According to the measurement principle of thrombus elasticity, a kind of supporting structure for the thromboelastography is designed. A kind of magnetic suspension support structure combined with active magnetic bearings and passive magnetic bearings is proposed. The radial suspension of the magnetic bearings relies on the magnetic force provided by the permanent magnets. The axial suspension is achieved by the electromagnetic force provided by the electromagnet. The electromagnetic simulation of Ansoft Maxwell is applied to optimize the structure of the radial magnetic bearing. By Simulink simulation, the appropriate PID parameters are found, and make the system's control work well. Finally, a magnetic suspension support structure with simple structure, small size and low cost is obtained, of which the radial bearing stiffness is $12.9653 \mathrm{~N} / \mathrm{mm}$ and the axial bearing of which the adjustment time of step response is $0.6 \mathrm{~s}$. This structure satisfies the measurement of thrombus elasticity.
\end{abstract}

\section{Introduction}

Thromboelastography (TEG) was invented by Dr. Hartert in 1948 and is mainly used for the detection of blood coagulation state ${ }^{[1,2]}$. Thromboelastography could diagnose coagulation function by measuring the viscosity of whole blood samples. The physical characteristics of blood clots (formation rate, clot strength and stability) determine whether the coagulation function is abnormal. As an important indicator of Clinical Hemorheology, thrombus graph is mainly used for the dynamic detection of coagulation function. Thrombus graph could be drawn by measuring the change of blood viscous torque during coagulation. Since the measurement of the viscous torque of blood belonged to the field of micro torque measurement, the measuring instrument required a friction free or only slight friction support structure. Magnetic suspension bearing is a kind of high performance supporting structure, which has the characteristics of non-contact, frictionless and non-lubricated ${ }^{[3,4,5,6,7]}$. In this paper, a new kind of support structure is designed, which is a new type of magnetic suspension bearing and combines the advantages of active magnetic bearings and passive magnetic bearings effectively. Support structure of thromboelastography

The structure diagram of the thromboelastography is shown in Fig. 1. The working principle is: $0.36 \mathrm{~mL}$ of whole blood is added to the test cup; heats test cup to keep the blood temperature at $37{ }^{\circ} \mathrm{C}$, the test cup is rotated at $\pm 4^{\circ} 45^{\prime}$ and at a rate of one week every $10 \mathrm{~s}$ ( $1 \mathrm{~s}$ at each boundary); blood gradually coagulates to form thrombus; the test cup lid, which placed in the blood specimen test cup, is affected by the viscous torque of the blood, with the left and right rotation; when the viscous torque of the blood and the reaction torque provided by the hairspring is equal, the shaft stops rotating, and the thrombus graph can be drawn by measuring the change of the rotation angle of the shaft during the coagulation process ${ }^{[2]}$. The support structure of the thrombus graph instrument is a magnetic suspension bearing combined with the active and the passive. The axis of the magnetic bearing is composed of differential electromagnet and thrust plate, which is suspended by electromagnetic force generated by electromagnet. The radial of the magnetic bearing is mainly composed of inner and outer magnetic rings with coaxial matching, which is suspended by the repulsion force generated by the inner and outer magnetic rings. 


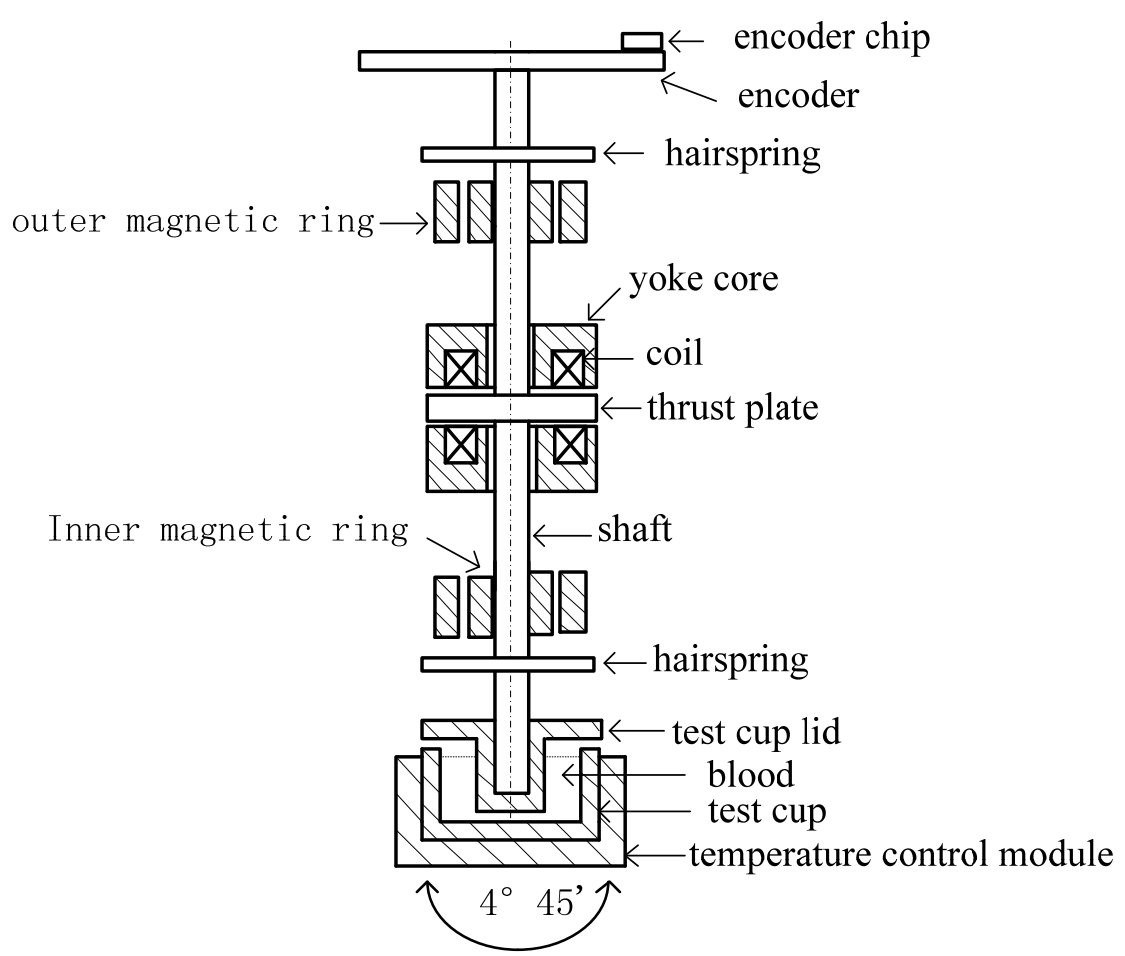

Fig. 1 Structure diagram

The radial direction of magnetic suspension bearing mainly depends on the magnetic force produced by permanent magnet. Magnetic force can be either repulsive or attractive, but the stability of repulsive system is better than that of ${ }^{[7]}$. When the rotating shaft is disturbed by the external force, the radial position of the rotating shaft is changed. The air gap in the inner magnetic ring and the outer ring is changed accordingly. The repulsive force of the small work air gap is greater than the repulsion of the large work air gap. In this case, the axis of rotation is affected by an opposing force, and finally becomes a balance state. However, due to the inherent damping of the passive magnetic bearing is very low ${ }^{[3]}$, it will take a long time for the bearing system to stabilize. Due to one end of the shaft immersed in the blood, the bearing system is added with a certain damping provide by the blood, and can be more stable down.

The axis of the magnetic bearing is applied to the differential electromagnet structure, which uses the electromagnetic force to control the position of the thrust plate to keep the shaft unchanged. When the external force acts on the rotating shaft to change the position of the thrust plate, the controller changes the coil current on the left and right to produce reaction force to make the thrust plate back to the balance position. The static stiffness, dynamic stiffness and damping of the active bearing can be adjusted by changing the control parameters. Therefore, the good control system can be achieved by selecting good control parameters ${ }^{[5,7]}$.

\section{Structure Analysis of Radial Passive Magnetic Bearing}

Fig. 2 and Fig. 3 are sectional models of a single ended radial permanent magnetic bearing, in which Fig. 2 is an axial magnetized structure model and Fig. 3 is a radial magnetizing structural model. The axial width of the inner and outer magnetic rings is denoted by 1 and $L$, respectively. The radial width of the inner and outer magnetic rings is denoted by $h$ and $\mathrm{H}$, respectively. The gap between the inner and outer magnetic rings is denoted by $\delta$. When the axial width of the inner and outer magnetic rings is closer, the bearing stiffness is larger. The width of the inner and outer magnetic rings is selected as the same $(1=\mathrm{L}){ }^{[7]}$. When $1=\mathrm{L}$, with the increase of 1 , permanent bearing load increases is not obvious ${ }^{[7]}$. Given the overall structure size, $\mathrm{l}=\mathrm{L}=6 \mathrm{~mm}$ is selected. The outer diameter of the shaft is fixed at $6 \mathrm{~mm}$, so that the inner diameter of the inner magnetic ring is limited to $6 \mathrm{~mm}$. The inner diameter of the outer ring is $28 \mathrm{~mm}$, so the outer diameter of the outer ring is limited to $28 \mathrm{~mm}$. 
The size of the inner and outer magnetic rings can be adjusted in the limited range so as to maximize the bearing stiffness of the radial permanent magnetic bearing.

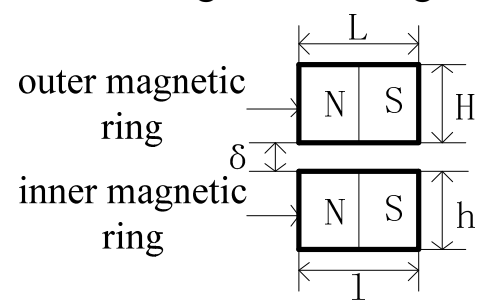

Fig. 2 Axial magnetization of single-end radial permanent magnet bearing

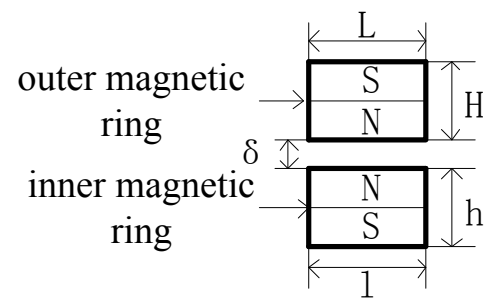

Fig. 3 Radial magnetization of single-end radial permanent magnet bearing

When the gap between the inner and outer magnetic rings is $\delta=1 \mathrm{~mm}$ and $\delta=2 \mathrm{~mm}$, the radial bearing stiffness of the radial permanent magnet bearing is analyzed by Ansoft Maxwell. When the gap $\delta=1 \mathrm{~mm}$, the center position of the inner and outer magnetic rings is adjusted to $0.2 \mathrm{~mm}, 0.4 \mathrm{~mm}$, $0.6 \mathrm{~mm}$ and $0.8 \mathrm{~mm}$ respectively, to determine the radial magnetic field force of inner magnetic ring. Similarly, when the gap $\delta=2 \mathrm{~mm}$, adjust the center offset distance to $0.4 \mathrm{~mm}, 0.8, \mathrm{~mm}, 1.2 \mathrm{~mm}, 1.6 \mathrm{~mm}$ respectively, to determine the radial magnetic field force. The simulation results are shown in Fig. 4. According to the simulation results, the smaller the gap, the greater the radial load stiffness, so $\delta=1 \mathrm{~mm}$ is taken.

There are two kinds of magnetizing methods for ring permanent magnets ${ }^{[6]}$, such as radial magnetization and bearing magnetization, which is shown in Fig. 2 and Fig. 3, respectively. The radial bearing stiffness of magnetic bearings under different magnetizing modes is analyzed by finite element simulation software. According to the simulation results of Fig. 5, due to the radial bearing stiffness of axial magnetization is higher, axial magnetization is adopted. In practice, the permanent magnet magnetization technology is easier to achieve ${ }^{[6]}$.

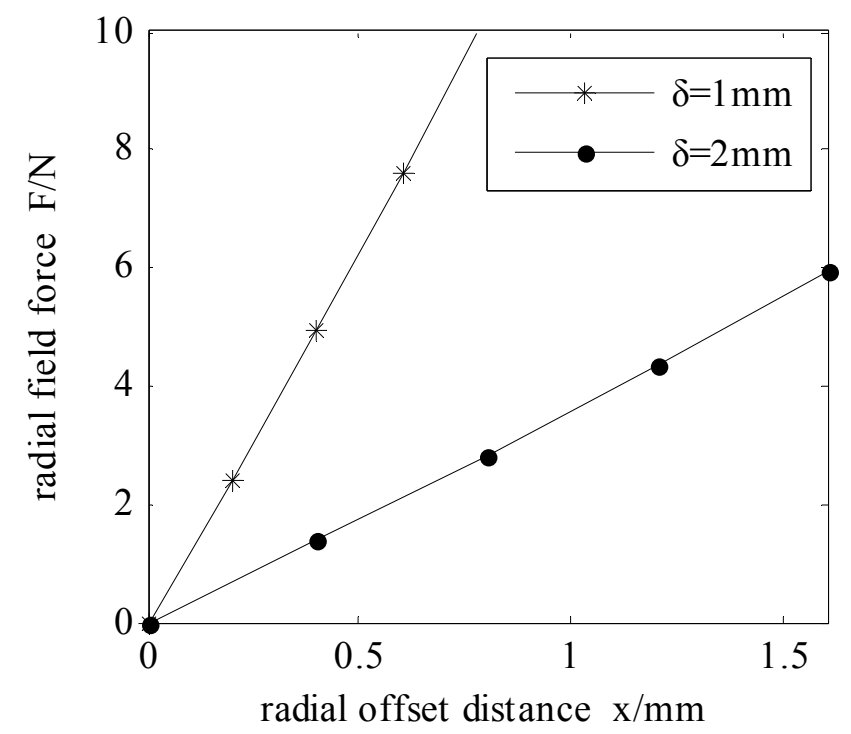

Fig. 4 when the gap is $1 \mathrm{~mm}$ and $2 \mathrm{~mm}$, the relationship between radial force and radial offset distance 


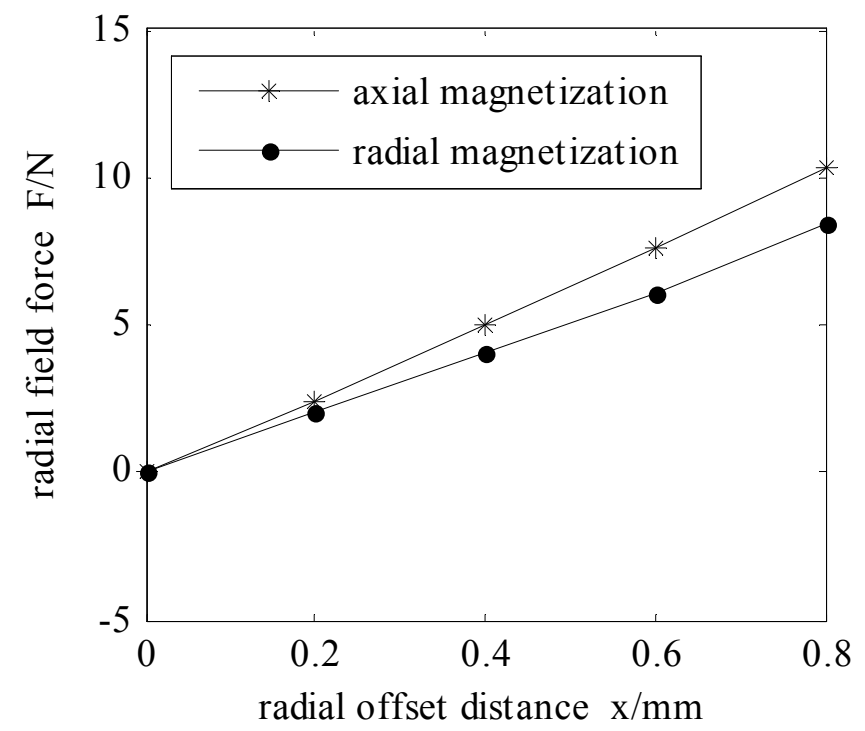

Fig. 5 The relationship between radial force and magnetizing method

\section{Structural Analysis of Axial Active Magnetic Bearings}

As shown in Fig. 1, the axial magnetic suspension bearings adopt the active magnetic suspension mode. When the shaft is subjected to an upward disturbing force, the thrust plate will deviate from the equilibrium position and move upward displacement $\delta$, resulting in the lower air gap increases to $\delta_{0}+\delta$, the upper air gap is reduced to $\delta_{0}-\delta$. At this time, the controller generates a control current $i$ to increase the suction force of the lower electromagnet, and reduces the suction of the upper electromagnet ${ }^{[4,5]}$. The lower electromagnetic force is $F_{1}$, and the upper electromagnetic force is $F_{2}$.

$$
\begin{aligned}
& F_{1}=\frac{\mu_{0} A n^{2}\left(i_{0}+i\right)^{2}}{4\left(\delta_{0}+\delta\right)^{2}} \\
& F_{2}=\frac{\mu_{0} A n^{2}\left(i_{0}-i\right)^{2}}{4\left(\delta_{0}-\delta\right)^{2}}
\end{aligned}
$$

The meaning of each parameter in the function is $: \mu_{0}$ : vacuum permeability, A: air gap area of axial magnetic bearings, $\mathrm{n}$ : number of turns of an electromagnet, $i_{0}$ : bias current of axial magnetic bearings in balanced state, $\delta_{0}$ : air gap of axial magnetic bearing in balance position.

The shaft is subjected to a downward force $F$ :

$$
F=F_{1}-F_{2}=\frac{\mu_{0} A n^{2}}{4}\left[\frac{\left(i_{0}+i\right)^{2}}{\left(\delta_{0}+\delta\right)^{2}}-\frac{\left(i_{0}-i\right)^{2}}{\left(\delta_{0}-\delta\right)^{2}}\right]
$$

The resultant $\mathrm{F}$ is nonlinear with the control current and the axial offset distance. In order to simplify the control, linearization is performed at the equilibrium position ${ }^{[3,4,5]}$. The electromagnetic force equation of the magnetic bearing is obtained.

$$
F=-K_{\delta} \delta+K_{i} i
$$

$K_{\delta}=\frac{\mu_{0} A n^{2} i^{2}}{\delta^{3}}$ is the displacement stiffness. $K_{i}=\frac{\mu_{0} A n^{2} i}{\delta^{2}}$ is the current stiffness. If the axial offset distance is set to the same direction as the electromagnetic force.

$$
F=K_{\delta} \delta+K_{i} i
$$

Since equation (5) is a linearized model obtained within a small deviation range, the accuracy of the electromagnetic force equation decreases as the distance of the offset equilibrium position increases. The simulation results of electromagnetic force and displacement are shown in Fig. 6 . With 
the increase of displacement, some nonlinear relation between electromagnetic force and displacement is found.

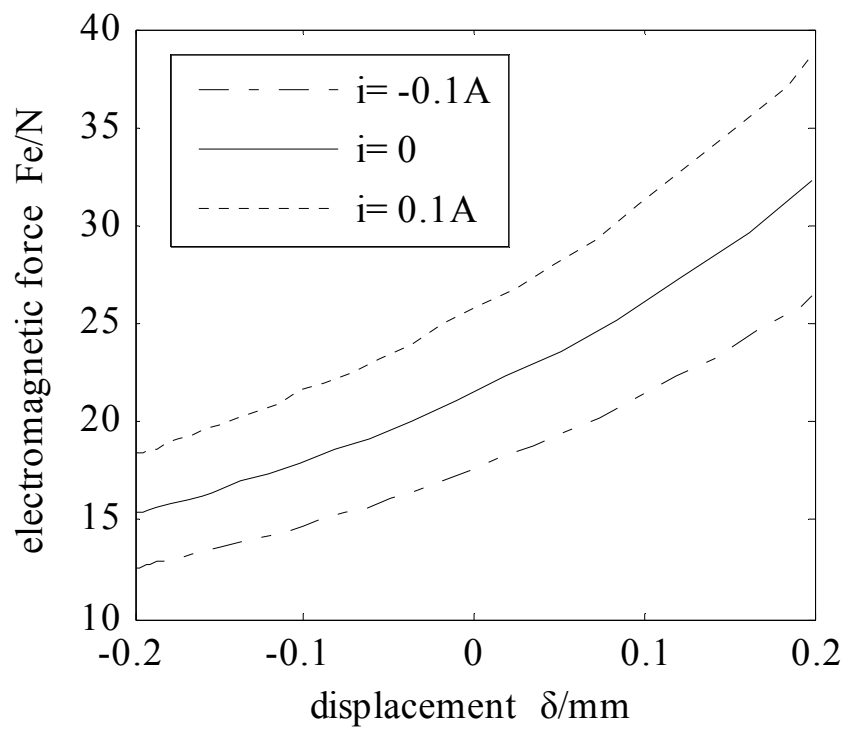

Fig. 6 The relationship between electromagnetic force and displacement

According to equation (5) and Newton's law of motion in the $\delta$ direction, a mechanical equation is obtained.

$$
m \delta=K_{\delta} \delta+K_{i} i
$$

Make a Laplace change to the equation (5). The open-loop transfer function is set up with $I(S)$ as input and $\Delta(S)$ as output.

$$
G(S)=\frac{\Delta(S)}{I(S)}=\frac{108.6}{200 S^{2}-108.6}
$$

Because the open-loop system has positive real roots, the system is unstable. Closed-loop control system can be obtained by adding feedback links and PID control to the open-loop system, is shown in Fig. 7.

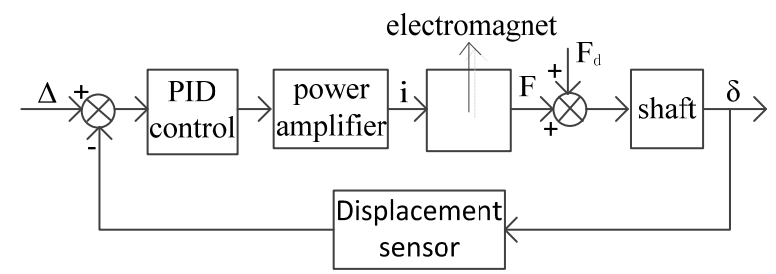

Fig. 7 Diagram of the control principle of the axial magnetic system

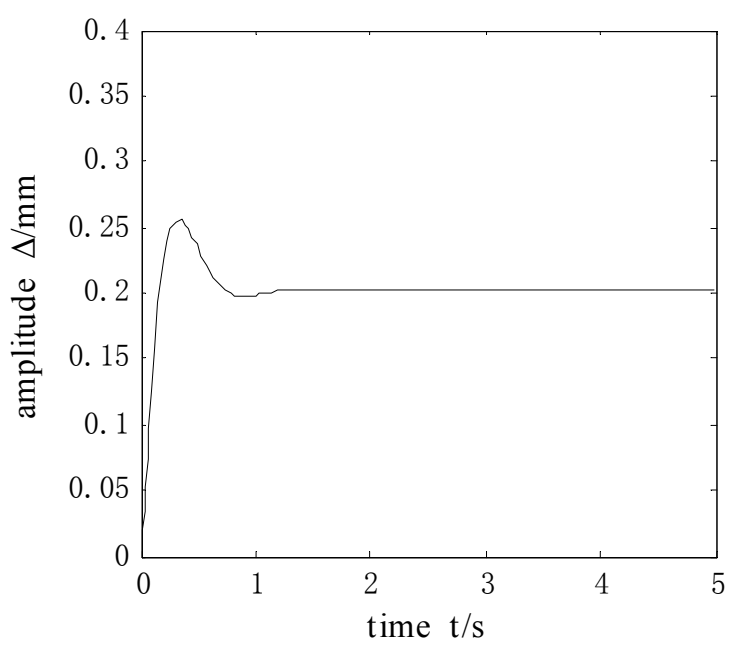

Fig. 8 Step response curve 
The appropriate control system can be obtained by adjusting the PID parameter, whose step response curve is shown in Fig. 8. As can be seen from the Fig. 8, the system overshoot is $27 \%$, the adjustment time is $0.6 \mathrm{~s}$.

\section{Summary}

The support structure is designed for the thromboelastography, which is a combination of active and passive magnetic bearings. The structure optimization of radial magnetic bearing is carried out by finite element simulation. The control effect of axial magnetic suspension bearing is verified by Simulink simulation. Because the measurement method of thrombus elasticity provides some damping for the radial direction of the magnetic levitation support structure, the anti-jamming ability of the system is improved. Finally, a support structure that meets the requirements of thrombus elasticity measurement is obtained.

\section{References}

[1]. Hartert H. Blutgerinnungsstudien mit der Thrombelastographie, einem neuen Untersuchungsverfahren. J Mol Med-jmm. Vol. 26(1948) No. 37, p.577-583.

[2]. Mallett SV, Cox DJ. Thromboelastography. British Journal of Anaesthesia. Vol. 63(1992), p.307-313.

[3]. XU Chang, ZHNAG Kai and ZHAO Lei. Magnetic suspension bearings-theory design and rotating machinery applications. Machinery Industry Press, 2007, p.7-38.

[4]. Feng Heng: Simulation Study on Active Magnetic Bearing System. Master, Nanjing University of Aeronautics and Astronautics, China, 2010. p. 1-27.

[5]. Shi Wenjuan: Study on Active Magnetic Bearing Control System Based on Arm. Master, Sichuan University, China, 2006. p. 1-27.

[6]. Wei Yong, Zhang Dawei. Permanent magnet magnetic bearing. Bearing. Vol. 8(2004), p.73-75.

[7]. Yang Haiyu, Chen Long. The role of passive magnetic bearing in magnetic suspension technology. Mechanical Engineering \& Automation. Vol. 4(2005), p.124-126. 